\title{
A Meta-Analysis of the Short- and Long-Term Results of Randomized Con- trolled Trials That Compared Laparoscopy-Assisted and Conventional Open Surgery for Colorectal Cancer
}

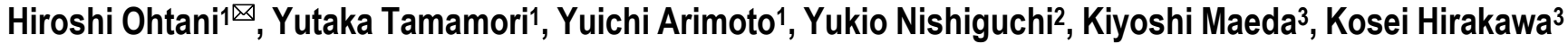 \\ 1. Department of Surgery, Osaka City Sumiyoshi Hospital \\ 2. Department of Surgery, Osaka City General Hospital \\ 3. Department of Surgical Oncology, Osaka City University Graduate School of Medicine
}

$\triangle$ Corresponding author: Hiroshi Ohtani, Department of Surgery, Osaka City Sumiyoshi Hospital, 1-2-16, Higashi-Kagaya, Suminoe-ku, Osaka 559-0012, Japan. Tel: +81-6-6681-1000; FAX: +81-6-6686-1547; E-mail: m5051923@msic.med.osaka-cu.ac.jp

(C) Ivyspring International Publisher. This is an open-access article distributed under the terms of the Creative Commons License (http://creativecommons.org/ licenses/by-nc-nd/3.0/). Reproduction is permitted for personal, noncommercial use, provided that the article is in whole, unmodified, and properly cited.

Received: 2011.05.23; Accepted: 2011.07.28; Published: 2011.08.01

\begin{abstract}
Purpose: We conducted a meta-analysis to evaluate and compare the short- and long-term results of laparoscopic colorectal surgery (LCRS) and conventional open surgery (OCRS) for colorectal cancer (CRC).

Methods: We searched relevant papers published between January 1990 and May 2011. We analyzed the outcomes of each type of surgery over the short- and long-term periods. Results: In the short-term period, we found no significant differences in overall perioperative complications and anastomotic leakage between LCRS and OCRS groups. We found no significant differences in overall, distant, local and wound-site recurrence, overall mortality, 3 and 5 year disease-free survival rate, and cancer-related mortality between the 2 groups.

Conclusions: LCRS has the benefits of reducing intraoperative blood loss, earlier resumption of oral intake, and shorter duration of hospital stay in the short-term. The long-term outcomes of LCRS seem to be similar to those of OCRS.
\end{abstract}

Key words: meta-analysis, laparoscopy-assisted colorectal surgery, colorectal cancer

\section{Introduction}

Colorectal cancer (CRC) is the fourth leading cause of cancer-specific mortality worldwide, with 610,000 related deaths each year ${ }^{1}$. CRC is the fourth most common form of cancer in the United States ${ }^{2}$ and the third leading cause of cancer-related death in the Western world $^{3}$. Surgery is the only curative treatment for CRC. Laparoscopic resection for CRC was first described in $1991^{4}$ and has since been widely applied by surgeons to treat patients with CRC.

Several articles have reported the short-term advantages of laparoscopic colorectal surgery (LCRS) over conventional open colorectal surgery (OCRS) and have concluded that laparoscopic surgery causes less pain, results in better pulmonary function, shorter duration of postoperative ileus, less fatigue, and a better quality of life ${ }^{5-7}$. However, the value of laparoscopic colorectal surgery has remained controversial because the long-term outcomes have not been clarified. The long-term results of colorectal surgery, such as tumor recurrence rate, disease-free survival rate, and mortality rate, have been gradually published ${ }^{8-10}$. Several randomized control trials (RCTs) that com- 
pare LCRS with OCRS have been conducted $^{8-30}$. Therefore, we conducted a meta-analysis of the data from these RCTs and compared the outcomes of LCRS and OCRS by considering several factors listed below. In addition, we selected the RCTs for which the follow-up period was at least 3 years to evaluate the long-term outcomes of LCRS.

\section{Materials and methods}

To identify papers relevant to our study we searched through the major medical databases such as MEDLINE, EMBASE, Science Citation Index, and Cochrane Controlled Trial Register for studies published between January 1990 and May 2011. The following search terms were used: "laparoscopy," "laparoscopy-assisted," "surgery," "colorectal cancer," and all related articles. Furthermore, we limited our literature search to those studies that involved a follow-up period of 3 or more years to examine the long-term outcomes of LCRS. We treated studies that are part of a series or studies described by the same author as a single study. Most appropriate data of a series of studies were used for this meta-analysis.

Three researchers (H.O., Y.T., and K.H.) extracted data from each article by using a structured sheet and entered the data into a database. Because this analysis was performed by the principle of intention-to-treat ${ }^{31}$, all patients converted from the laparoscopic group to the conventional open surgery group remained in the laparoscopic group for analysis. We conducted a meta-analysis for the short- and long-term. For the short-term analysis, we collected data on the duration of the operation, estimated blood loss, number of patients requiring transfusion, number of harvested lymph nodes, time required for resumption of oral intake, duration of hospital stay, length of operation wound, complications, and perioperative mortality. For the long-term analysis, we used data on the rate of tumor recurrence, disease-free survival rate, and mortality. If necessary, we contacted the authors of the original studies to receive further information.

\section{Statistical analysis}

Weighted mean differences (WMDs) and odds ratios (ORs) were used for the analysis of continuous and dichotomous variables, respectively. Random-effects models were used to identify heterogeneity between the studies ${ }^{32}$ and the degree of heterogeneity was assessed using the $\chi^{2}$ test. The confidence interval (CI) was established at $95 \%$ and $p$ values of less than 0.05 were considered to indicate statistical significance. Statistical analyses were performed using the Review Manager (RevMan) software version
5.0.25 provided by the Cochrane Collaboration, Copenhagen, Denmark.

\section{Results}

We identified 12 papers reporting RCTs that compared LCRS and OCRS for colorectal cancer8-24. The characteristics of each RCT are presented in Table 1. Our meta-analysis included 4458 patients with colorectal cancer; of these, 2375 had undergone LCRS, and 2083, OCRS. The results of the short- and long-term are shown in Fig. 1 and Fig. 2, respectively. The outcomes of LCRS and OCRS in the short- and long-term are reported below.

\section{Short-term Outcomes}

The operative duration for LCRS was significantly longer than for OCRS, i.e., by $39.32 \mathrm{~min}$ (WMD $=39.32 ; 95 \% \mathrm{CI}=30.72-47.91 ; \mathrm{p}<0.00001)$. Eleven of the 12 RCTs included data on operative duration, and the 11 RCTs indicated that the duration of operations using LCRS was significantly longer than that of operations using OCRS. Blood loss in patients who underwent LCRS was significantly lesser than patients in those who underwent OCRS, by an average volume of $133.05 \mathrm{ml}(\mathrm{WMD}=-133.05 ; 95 \% \mathrm{CI}=-201.30$ to $-64.81 ; \mathrm{p}=0.0001)$. We found no significant differences between patients who underwent LCRS and those that had OCRS for the number of transfused patients or the number of dissected lymph nodes. Patients in the LCRS group resumed oral intake on an average of 1.08 days sooner than did patients in the OCRS group, and the difference was significant (WMD $=-1.08 ; 95 \% \mathrm{CI}=-1.36$ to $-0.80 ; \mathrm{p}<0.00001$ ). The duration of hospital stay was significantly shorter by an average of 2.80 days for patients in the LCRS group than for those in the OCRS group (WMD = $-2.80 ; 95 \% \mathrm{CI}=-4.78$ to $-0.81 ; \mathrm{p}=0.006$ ). The average length of the wound caused by each operation was significantly shorter by $10.97 \mathrm{~cm}$ in the LCRS group than in the OCRS group $(\mathrm{WMD}=-10.97 ; 95 \% \mathrm{CI}=$ -14.37 to $-7.57 ; \mathrm{p}<0.00001)$. Differences in overall perioperative complications and anastomotic leakage between the LCRS group and the OCRS group were insignificant for treatment of the colorectal cancer. We also found no significant differences in perioperative mortality between the surgery groups when we pooled data for treatment of the colorectal cancer.

\section{Long-term Outcomes}

The rate of wound-site recurrence for patients in the LCRS group was significantly higher than for those in the OCRS group in our analysis of the pooled data for CRC treatment $(\mathrm{OR}=2.87 ; 95 \% \mathrm{CI}=$ $1.08-7.68 ; \mathrm{p}=0.04)$. Restricting wound-recurrence to 
isolated abdominal-wall recurrences, in the absence of recurrent disease elsewhere, the differences between the groups was insignificant $(p=0.09)$. Our analysis of the local and distant metastasis recurrence between the LCRS group and the OCRS group for treatment of the colorectal cancer indicated no significant difference. There was also no significant difference between the surgery groups for the overall recurrence of tumors.

We found no significant differences in the 3- and 5-year disease-free survival rates between patients who underwent LCRS and those who underwent OCRS.
There was no significant difference between the LCRS and OCRS groups for cancer-related mortality for treatment of the colorectal cancer. Likewise, there was no significant difference in overall mortality between the LCRS and OCRS groups.

\section{Heterogeneity}

In the short-term period, significant heterogeneity was detected between studies with respect to the following 4 factors: intraoperative blood loss, duration of hospital stay, length of operation wound, and overall complications. In the long-term period, no significant heterogeneity was detected between studies.

Table 1. Characteristics of the randomized clinical trials

\begin{tabular}{|c|c|c|c|c|c|c|c|}
\hline \multirow[t]{2}{*}{ authors } & \multirow[t]{2}{*}{ Year } & \multirow{2}{*}{$\begin{array}{l}\text { number of ref- } \\
\text { erence }\end{array}$} & \multirow{2}{*}{$\begin{array}{l}\text { institutions of } \\
\text { the study }\end{array}$} & \multicolumn{2}{|c|}{ Study size (n) } & \multirow[t]{2}{*}{ lesional site } & \multirow{2}{*}{$\begin{array}{l}\text { follow-up period } \\
\text { (months) }\end{array}$} \\
\hline & & & & LCRS & OCRS & & \\
\hline Araujo et al. & 2003 & 11 & single center & 13 & 15 & rectum & 47.2 months (mean) \\
\hline Braga at al. (colon) & 2010 & 12 & single center & 134 & 134 & colon & 73 months (median) \\
\hline $\begin{array}{l}\text { Braga at al. (rec- } \\
\text { tum) }\end{array}$ & 2007 & 13 & single center & 83 & 85 & rectum & $\begin{array}{l}53.6 \text { months (mean)/ } \\
54.2 \text { months (median) }\end{array}$ \\
\hline CLASICC & $\begin{array}{l}2010,2007, \\
2005\end{array}$ & $8,9,16$ & multicenter & 526 & 268 & colon or rectum & 56.3 months (median) \\
\hline COLOR & 2009, 2005 & 17,18 & multicenter & 534 & 542 & colon & 53 months (median) \\
\hline COST & 2004 & 19 & multicenter & 435 & 428 & colon & 4.4 years (median) \\
\hline Curet et al. & 2000 & 20 & single center & 25 & 18 & colon & 4.9 years (mean) \\
\hline Lacy & 2002 & 10 & single center & 111 & 108 & colon & 43 months (median) \\
\hline Leung & 2004 & 21 & 2 centers & 167 & 170 & colon or rectum & 51 months (median) \\
\hline Liang & 2006 & 22 & single center & 135 & 134 & colon & 40 months (median) \\
\hline Mirza et al. & 2008 & 23 & single center & 116 & 117 & colon or rectum & 48 months (median) \\
\hline Park et al. & 2009 & 24 & single center & 170 & 374 & rectum & 36 months (mean) \\
\hline
\end{tabular}

operative time

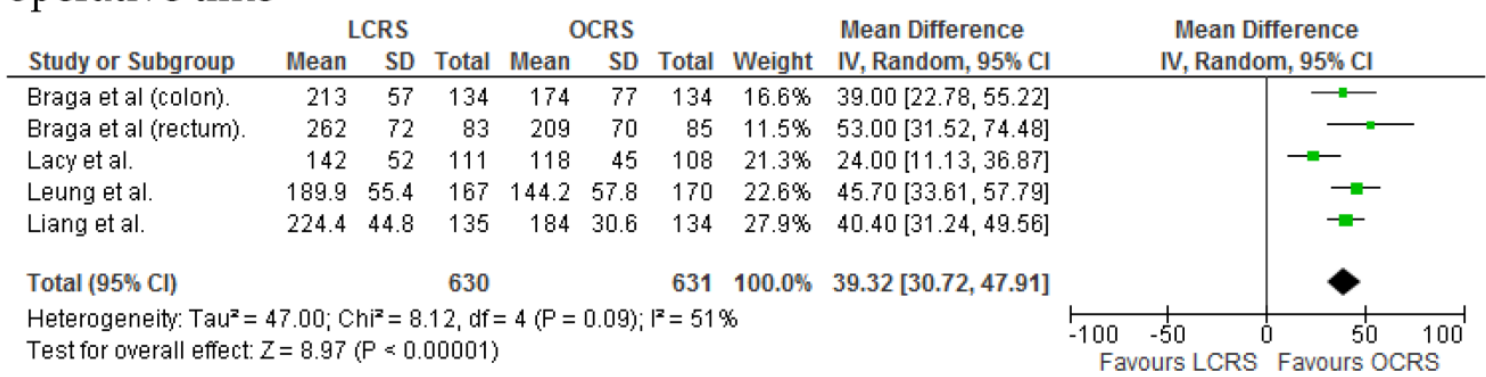

estimated blood loss

\begin{tabular}{|c|c|c|c|c|c|c|c|c|c|c|c|}
\hline \multirow[b]{2}{*}{ Study or Subgroup } & \multicolumn{3}{|c|}{ LCRS } & \multicolumn{3}{|c|}{ OCRS } & \multicolumn{3}{|c|}{ Mean Difference } & \multirow{2}{*}{\multicolumn{2}{|c|}{$\begin{array}{c}\text { Mean Difference } \\
\text { IV, Random, } 95 \% \mathrm{Cl}\end{array}$}} \\
\hline & Mean & SD & Total & Mean & SD & Total & Weight & IV, Random, $95 \% \mathrm{Cl}$ & & & \\
\hline Braga et al (colon). & 46 & 130 & 134 & 127 & 265 & 134 & $25.4 \%$ & $-81.00[-130.98,-31.02]$ & & $\longrightarrow-$ & \\
\hline Braga et al (rectum). & 213 & 236 & 83 & 396 & 367 & 85 & $19.0 \%$ & $-183.00[-276.09,-89.91]$ & $\longleftarrow$ & & \\
\hline Lacy et al. & 105 & 99 & 111 & 193 & 212 & 108 & $26.2 \%$ & $-88.00[-132.02,-43.98]$ & & $\longrightarrow$ & \\
\hline Liang et al. & 54 & 12 & 135 & 240 & 34 & 134 & $29.4 \%$ & $-186.00[-192.10,-179.90]$ & a & & \\
\hline Total $(95 \% \mathrm{Cl})$ & & & 463 & & & 461 & $100.0 \%$ & $-133.05[-201.30,-64.81]$ & & & \\
\hline $\begin{array}{l}\text { Heterogeneity: } \operatorname{Tau}^{2}= \\
\text { Test for overall effect: }\end{array}$ & $\begin{array}{l}120.23 \\
=3.82\end{array}$ & $\begin{array}{l}\mathrm{Chi}^{2}= \\
(\mathrm{P}=0 .\end{array}$ & $\begin{array}{l}=34.81 \\
.0001)\end{array}$ & $d f=3$ & $(P \leq 0$ & .00001 & $;\left.\right|^{2}=919$ & & $\begin{array}{l}+200 \\
\text { Favo }\end{array}$ & $\begin{array}{l}-100 \\
\text { urs LCRS }\end{array}$ & $\begin{array}{l}100 \\
\text { Favours OCRS }\end{array}$ \\
\hline
\end{tabular}


number of transfused patients

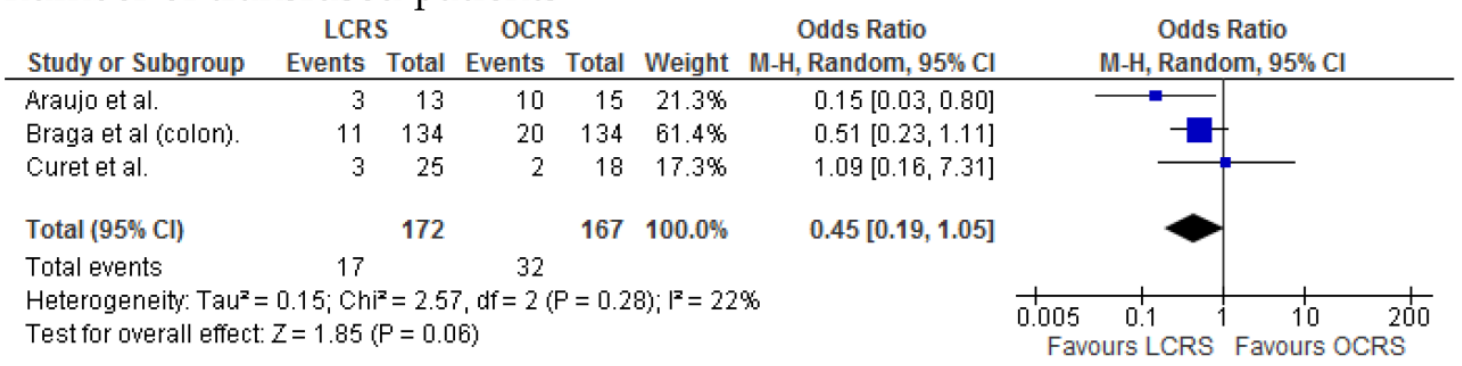

number of dissected lymph nodes

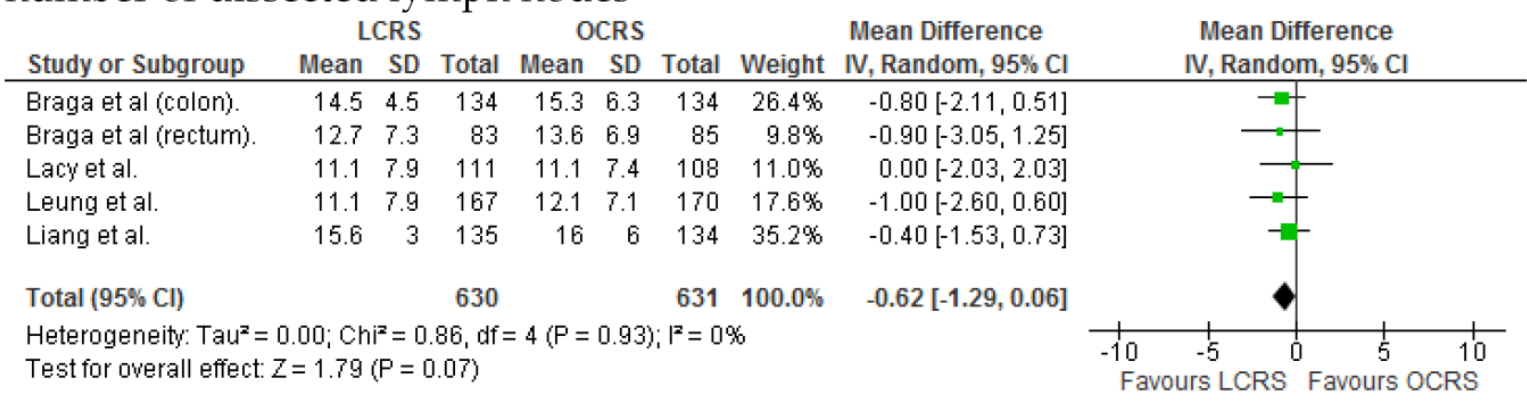

\section{hospital stay}

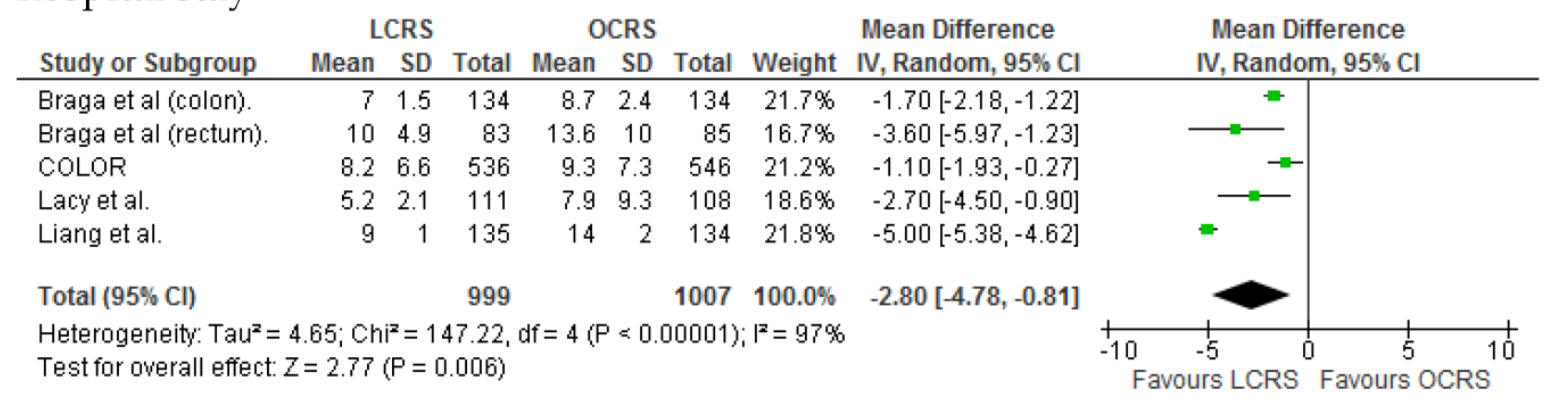

time to oral intake

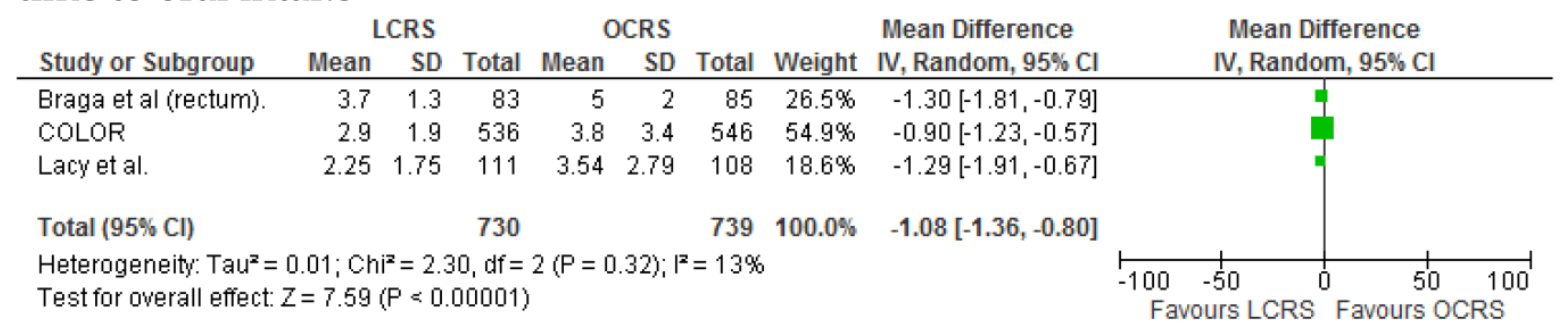

\section{length of operation wound}

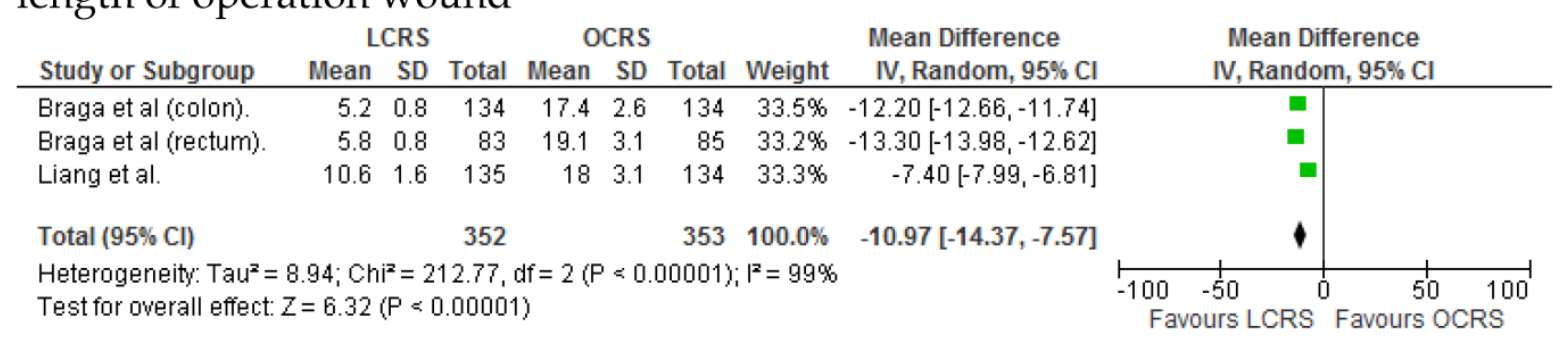


overall complication

LCRS

OCRS

Odds Ratio

Odds Ratio

Study or Subgroup Events Total Events Total Weight M-H, Random, $95 \% \mathrm{Cl}$

g 13

$\begin{array}{lll}7 & 15 & 2.1 \%\end{array}$

$2.57[0.54,12.17]$

Braga et al (colon).

$20 \quad 134$

$\begin{array}{lll}33 & 134 & 8.7 \%\end{array}$

$0.54[0.29,0.99]$

Braga et al (rectum)

$24 \quad 83$

$34 \quad 85 \quad 8.2 \%$

CLASICC

$85 \quad 268 \quad 15.6 \%$

COLOR

CosT

$\begin{array}{rr}111 & 534 \\ 92 & 435\end{array}$

$110 \quad 542 \quad 16.1 \%$

$0.61[0.32,1.16]$

Curet et al

Lacy et al.

Leung et al.

Liang et al.

225

$\begin{array}{lll}85 & 428 & 15.2 \%\end{array}$

$1.05[0.76,1.43]$

$1.03[0.77,1.39]$

$1.08[0.78,1.51]$

$0.43[0.06,2.92]$

$0.30[0.15,0.62]$

$\begin{array}{lllll}12 & 111 & 31 & 108 & 7.0 \%\end{array}$

Parket al.

$20 \quad 135$

$0.87[0.53,1.43]$

$0.63[0.34,1.18]$

Total $(95 \% \mathrm{Cl})$

$10 \quad 170$

$29 \quad 134 \quad 8.5 \%$

$1.24[0.56,2.74]$

Total events

2333

$2276 \quad 100.0 \%$

$0.83[0.66,1.05]$

Heterogeneity: $\operatorname{Tau}^{2}=0.07 ; \mathrm{Chi}^{2}=19.81, \mathrm{df}=10(\mathrm{P}=0.03) ; \mathrm{I}^{2}=50 \%$

Test for overall effect: $Z=1.57(P=0.12)$

M-H, Random, $95 \% \mathrm{Cl}$

anastomotic leakage

LCRS

OCRS

Odds Ratio

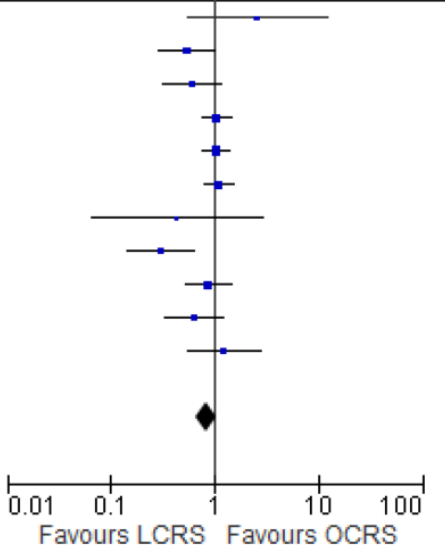

Favours LCRS Favours OCRS

Study or Subgroup Events Total Events Total Weight M-H, Random, 95\% Cl

Braga et al (colon).

Braga et al (rectum)

CLASICC

COLOR

Curet et al.

Lacy et al.

Leung et al.

Liang et al.

Park et al.

5 Total

$0.83[0.25,2.78]$

$0.90[0.33,2.46]$

$\begin{array}{lllll}8 & 83 & 9 & 85 & 13.3 \%\end{array}$

$\begin{array}{rrrrr}35 & 526 & 13 & 268 & 31.3 \% \\ 15 & 534 & 10 & 542 & 20.4 \%\end{array}$

0 25

$\begin{array}{rr}10 & 542 \\ 0 & 18\end{array}$

0 111

1167

$2108 \quad 1.4 \%$

$\begin{array}{lll}4 & 170 & 2.8 \%\end{array}$

2135

$4 \quad 134 \quad 4.6 \%$

$8 \quad 141$

$\begin{array}{lll}15 & 261 & 17.1 \%\end{array}$

Total $(95 \% \mathrm{Cl})$

1856

Total events

74 63

Heterogeneity: $\mathrm{Tau}^{2}=0.00 ; \mathrm{Ch}^{2}=5.45, \mathrm{df}=7(\mathrm{P}=0.61) ; \mathrm{I}^{2}=0 \%$

Test for overall effect: $Z=0.35(P=0.73)$

$1.40[0.73,2.69]$

$1.54[0.68,3.45]$

Not estimable

$0.19[0.01,4.03]$

$0.25[0.03,2.26]$

$0.49[0.09,2.71]$

$0.99[0.41,2.39]$

$1.07[0.74,1.54]$

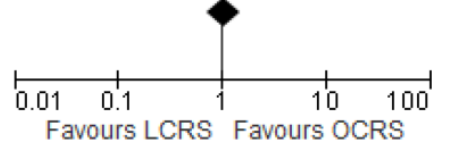

perioperative mortality

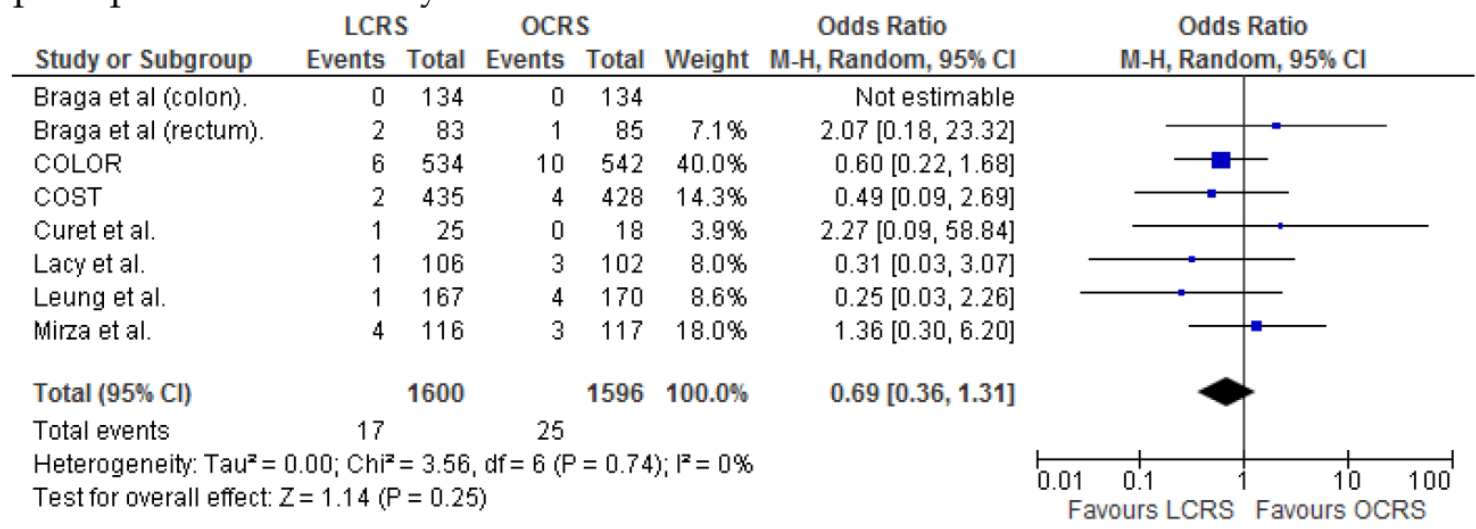

Fig. 1 Meta-analysis of the short-term period for colorectal cancer 


\section{overall recurrence}

LCRS OCRS Odds Ratio Odds Ratio

Study or Subgroup Events Total Events Total Weight M-H, Random, $95 \% \mathrm{Cl}$ $\mathrm{M}-\mathrm{H}$, Random, $95 \% \mathrm{Cl}$

\begin{tabular}{|c|c|c|c|c|c|c|c|c|c|}
\hline Araujo et al. & 0 & 13 & 2 & 15 & $0.2 \%$ & $0.20[0.01,4.57]$ & $\longleftarrow$ & \multirow{2}{*}{$\rightarrow$} & \\
\hline Braga et al (colon). & 20 & 134 & 24 & 134 & $5.5 \%$ & $0.80[0.42,1.54]$ & & & \\
\hline Braga et al (rectum). & 4 & 83 & 5 & 85 & $1.3 \%$ & $0.81[0.21,3.13]$ & & & \\
\hline CLASICC & 124 & 526 & 59 & 268 & $18.7 \%$ & $1.09[0.77,1.55]$ & & & \\
\hline COLOR & 105 & 534 & 92 & 542 & $24.2 \%$ & $1.20[0.88,1.63]$ & & & 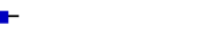 \\
\hline Cost & 76 & 435 & 84 & 428 & $19.6 \%$ & $0.87[0.61,1.22]$ & & & \\
\hline Curet et al. & 1 & 18 & 1 & 18 & $0.3 \%$ & $1.00[0.06,17.33]$ & & & \\
\hline Lacy et al. & 18 & 106 & 28 & 102 & $5.2 \%$ & $0.54[0.28,1.05]$ & & & \\
\hline Leung et al. & 37 & 167 & 30 & 170 & $8.0 \%$ & $1.33[0.78,2.27]$ & & & \\
\hline Liang et al. & 23 & 135 & 29 & 134 & $6.2 \%$ & $0.74[0.40,1.37]$ & & $\longrightarrow$ & \\
\hline Mirza et al. & 27 & 117 & 27 & 115 & $6.2 \%$ & $0.98[0.53,1.80]$ & & & \\
\hline Park et al. & 24 & 107 & 17 & 72 & $4.6 \%$ & $0.94[0.46,1.90]$ & & & \\
\hline Total $(95 \% \mathrm{Cl})$ & & 2375 & & 2083 & $100.0 \%$ & $0.98[0.84,1.14]$ & & 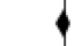 & 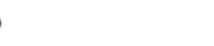 \\
\hline Total events & 459 & & 398 & & & & & & \\
\hline $\begin{array}{l}\text { Heterogeneity: } \operatorname{Tau}^{2}= \\
\text { Test for overall effect: }\end{array}$ & $\begin{array}{l}\text {; } \mathrm{Chi}^{2} \\
0.24(\mathrm{~F}\end{array}$ & $\begin{array}{l}=8.97, \\
=0.81\end{array}$ & $=11(\mathrm{~F}$ & $=0.62$ & $2) ;\left.\right|^{2}=0 \%$ & & \begin{tabular}{|lr}
0.01 & 0.1 \\
& Favours
\end{tabular} & ${ }^{1}{ }^{1}$ & $\begin{array}{c}10 \\
\text { Favours OCRS }\end{array}$ \\
\hline
\end{tabular}

\section{local recurrence}

LCRS OCRS Odds Ratio Odds Ratio

Study or Subgroup Events Total Events Total Weight M-H, Random, 95\% Cl

M-H, Random, $95 \% \mathrm{Cl}$

$\begin{array}{lrrrrr}\text { Araujo et al. } & 0 & 13 & 2 & 15 & 1.1 \% \\ \text { Braga et al (colon). } & 1 & 134 & 3 & 134 & 2.1 \% \\ \text { Braga et al (rectum). } & 3 & 83 & 4 & 85 & 4.5 \% \\ \text { CLASICC } & 45 & 526 & 21 & 268 & 28.9 \% \\ \text { COLOR } & 26 & 534 & 26 & 542 & 27.5 \% \\ \text { Lacy et al. } & 7 & 106 & 14 & 102 & 11.0 \% \\ \text { Leung et al. } & 11 & 167 & 7 & 170 & 10.6 \% \\ \text { Mirza et al. } & 4 & 117 & 11 & 115 & 7.4 \% \\ \text { Park et al. } & 6 & 107 & 5 & 72 & 6.9 \% \\ & & & & & \\ \text { Total (95\% Cl) } & & 1787 & & 1503 & 100.0 \% \\ \text { Total events } & 103 & & 93 & & \end{array}$

Heterogeneity: $\operatorname{Tau}^{2}=0.02 ; \mathrm{Chi}^{2}=8.73, \mathrm{df}=8(\mathrm{P}=0.37) ; \mathrm{I}^{2}=8 \%$

Test for overall effect: $Z=0.92(P=0.36)$

$0.20[0.01,4.57]$

$0.33[0.03,3.20]$

$0.76[0.16,3.50]$

$1.10[0.64,1.89]$

$1.02[0.58,1.77]$

$0.44[0.17,1.15]$

$1.64[0.62,4.34]$

$0.33[0.10,1.08]$

$0.80[0.23,2.71]$

$0.86[0.62,1.19]$

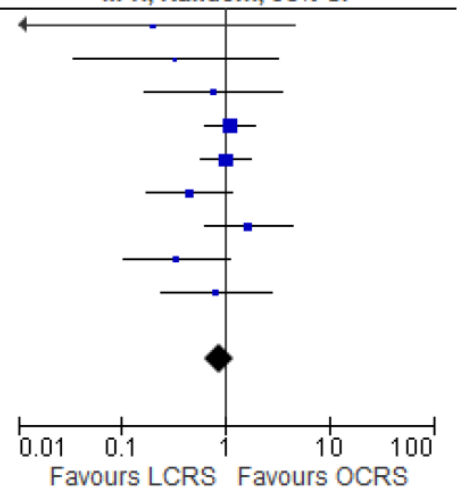

\section{distant metastasis}

LCRS OCRS Odds Ratio Odds Ratio

Study or Subgroup Events Total Events Total Weight M-H, Random, $95 \% \mathrm{Cl}$

\begin{tabular}{|c|c|c|c|c|c|}
\hline Araujo et al. & 0 & 13 & 0 & 15 & \\
\hline Braga et al (colon). & 19 & 134 & 21 & 134 & $8.9 \%$ \\
\hline Braga et al (rectum). & 1 & 83 & 1 & 85 & $0.5 \%$ \\
\hline CLASICC & 79 & 526 & 38 & 268 & $23.0 \%$ \\
\hline COLOR & 56 & 534 & 54 & 542 & $25.9 \%$ \\
\hline Lacy et al. & 7 & 106 & 9 & 102 & $3.8 \%$ \\
\hline Leung et al. & 30 & 167 & 26 & 170 & $12.2 \%$ \\
\hline Liang et al. & 22 & 135 & 28 & 134 & $10.6 \%$ \\
\hline Mirza et al. & 23 & 117 & 18 & 115 & $8.7 \%$ \\
\hline Park et al. & 18 & 107 & 12 & 72 & $6.3 \%$ \\
\hline Total $(95 \% \mathrm{Cl})$ & \multicolumn{3}{|c|}{1922} & 1637 & $100.0 \%$ \\
\hline Total events & \multicolumn{2}{|c|}{255} & \multicolumn{2}{|l|}{207} & \\
\hline \multicolumn{6}{|c|}{$\begin{array}{l}\text { Heterogeneity: } \operatorname{Tau}^{2}=0.00 ; \mathrm{Chi}^{2}=2.61, \mathrm{df}=8(P=0.96) ;\left.\right|^{2}=0 \% \\
\text { Test for overall effect: } Z=0.24(P=0.81)\end{array}$} \\
\hline
\end{tabular}

Not estimable

$0.89[0.45,1.74]$

$1.02[0.06,16.65]$

$1.07[0.70,1.63]$

$1.06[0.71,1.57]$

$0.73[0.26,2.04]$

$1.21[0.68,2.16]$

$0.74[0.40,1.37]$

$1.32[0.67,2.60]$

$1.01[0.45,2.25]$

$1.02[0.84,1.25]$

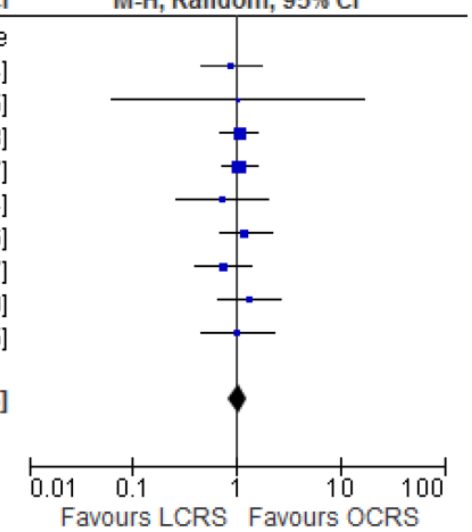




\section{wound-site recurrence}

LCRS

OCRS

Odds Ratio

Odds Ratio

Study or Subgroup Events Total Events Total Weight M-H, Random, $95 \% \mathrm{Cl}$

Araujo et al.

Braga et al (colon)

CLASICC

$\begin{array}{lr}0 & 13 \\ 0 & 134\end{array}$

015

Not estimable

Not estimable

COLOR

9 526

134

COST

7534

$\begin{array}{lll}1 & 26822.5 \%\end{array}$

Lacy et al.

Leung et al.

Liang et al.

Mirza et al.

2435

$2 \quad 542 \quad 38.9 \%$

$4.65[0.59,36.88]$

$3.59[0.74,17.34]$

$1.97[0.18,21.83]$

$2.91[0.12,72.38]$

Not estimable

o 167

o $102 \quad 9.4 \%$

$\begin{array}{ll}0 & 167 \\ 1 & 135\end{array}$

0 170

$12.5 \%$

$0.99[0.06,16.03]$

Not estimable

Total $(95 \% \mathrm{Cl})$

2167

0 115

$2.87[1.08,7.68]$

Total events $20 \quad 5$

Heterogeneity: $\operatorname{Tau}^{2}=0.00 ; \mathrm{Chi}^{2}=0.95, \mathrm{df}=4(\mathrm{P}=0.92) ; \mathrm{I}^{2}=0 \%$

Test for overall effect: $Z=2.10(P=0.04)$

M-H, Random, $95 \% \mathrm{Cl}$

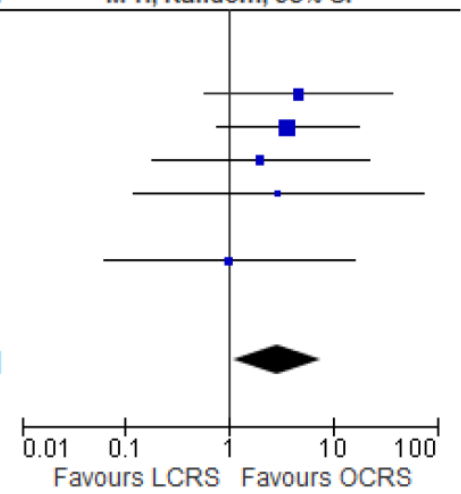

cancer-related mortality

LCRS

OCRS

Odds Ratio

Odds Ratio

Study or Subgroup Events Total Events Total Weight M-H, Random, 95\% Cl

\begin{tabular}{|c|c|c|c|c|c|}
\hline CLASICC & 94 & 526 & 50 & 268 & $25.7 \%$ \\
\hline COLOR & 58 & 534 & 69 & 542 & $26.4 \%$ \\
\hline cost & 48 & 435 & 61 & 428 & $23.8 \%$ \\
\hline Curet et al. & 5 & 25 & 6 & 18 & $3.1 \%$ \\
\hline Lacy et al. & 10 & 106 & 21 & 102 & $8.3 \%$ \\
\hline Leung et al. & 26 & 167 & 20 & 170 & $12.7 \%$ \\
\hline Total $(95 \% \mathrm{Cl})$ & \multicolumn{3}{|c|}{1793} & 1528 & $100.0 \%$ \\
\hline Total events & 241 & & 227 & & \\
\hline \multicolumn{6}{|c|}{$\begin{array}{l}\text { Heterogeneity: } \operatorname{Tau}^{2}=0.03 ; \mathrm{Chi}^{2}=6.89, \mathrm{df}=5(\mathrm{P}=0.23) ; \mathrm{I}^{2}=27 \% \\
\text { Test for overall effect: } Z=1.46(P=0.14)\end{array}$} \\
\hline
\end{tabular}

$0.95[0.65,1.39]$

$0.84[0.58,1.21]$

$0.75[0.50,1.12]$

$0.50[0.13,2.00]$

$0.40[0.18,0.90]$

$1.38[0.74,2.59]$

$0.83[0.65,1.07]$

Favours LCRS Favours OCRS

3-year disease-free interval

\begin{tabular}{|c|c|c|c|c|c|c|c|c|}
\hline \multirow[b]{2}{*}{ Study or Subgroup } & \multicolumn{2}{|c|}{ LCRS } & \multicolumn{2}{|c|}{ OCRS } & \multirow[b]{2}{*}{ Weight } & \multirow{2}{*}{$\begin{array}{c}\text { Odds Ratio } \\
\text { M-H, Random, } 95 \% \mathrm{Cl}\end{array}$} & \multirow{2}{*}{$\begin{array}{c}\text { Odds Ratio } \\
\text { M-H, Random, } 95 \% \mathrm{Cl}\end{array}$} & \\
\hline & Events & Total & Events & Total & & & & \\
\hline CLASICC & 284 & 428 & 143 & 211 & $35.3 \%$ & $0.94[0.66,1.33]$ & & \\
\hline COLOR & 396 & 534 & 413 & 542 & $57.0 \%$ & $0.90[0.68,1.18]$ & & \\
\hline Park et al. & 83 & 107 & 59 & 72 & $7.7 \%$ & $0.76[0.36,1.62]$ & & \\
\hline Total $(95 \% \mathrm{Cl})$ & & 1069 & & 825 & $100.0 \%$ & $0.90[0.73,1.11]$ & & \\
\hline Total events & 763 & & 615 & & & & & \\
\hline $\begin{array}{l}\text { Heterogeneity: Tau } \\
\text { Test for overall effect }\end{array}$ & $\begin{array}{l}0.00 ; \text { Chi } \\
\mathrm{Z}=0.99\end{array}$ & $\begin{array}{l}P=0.2 \\
P=0.3\end{array}$ & 4, $d f=2($ & $P=0.8$ & $9) ;\left.\right|^{2}=0 \%$ & & 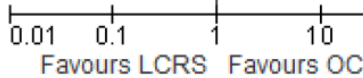 & $\begin{array}{l}100 \\
\mathrm{RS}\end{array}$ \\
\hline
\end{tabular}

\section{5-year disease-free interval}

LCRS OCRS

Odds Ratio

Odds Ratio

Study or Subgroup Events Total Events Total Weight M-H, Random, 95\% Cl

$\begin{array}{lrrrrr}\text { Braga et al (colon). } & 84 & 134 & 84 & 134 & 17.4 \% \\ \text { CoLoR } & 355 & 534 & 368 & 542 & 65.9 \% \\ \text { Leung et al. } & 126 & 167 & 133 & 170 & 16.6 \% \\ & & & & & \\ \text { Total (95\% Cl) } & & 835 & & \mathbf{8 4 6} & \mathbf{1 0 0 . 0 \%} \\ \text { Total events } & 565 & & 585 & & \end{array}$

Heterogeneity: $\operatorname{Tau}^{2}=0.00 ; \mathrm{Chi}^{2}=0.19, \mathrm{df}=2(\mathrm{P}=0.91) ; \mathrm{I}^{2}=0 \%$

Test for overall effect: $Z=0.65(P=0.52)$

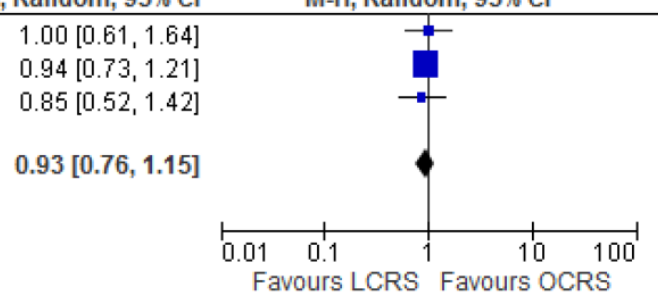




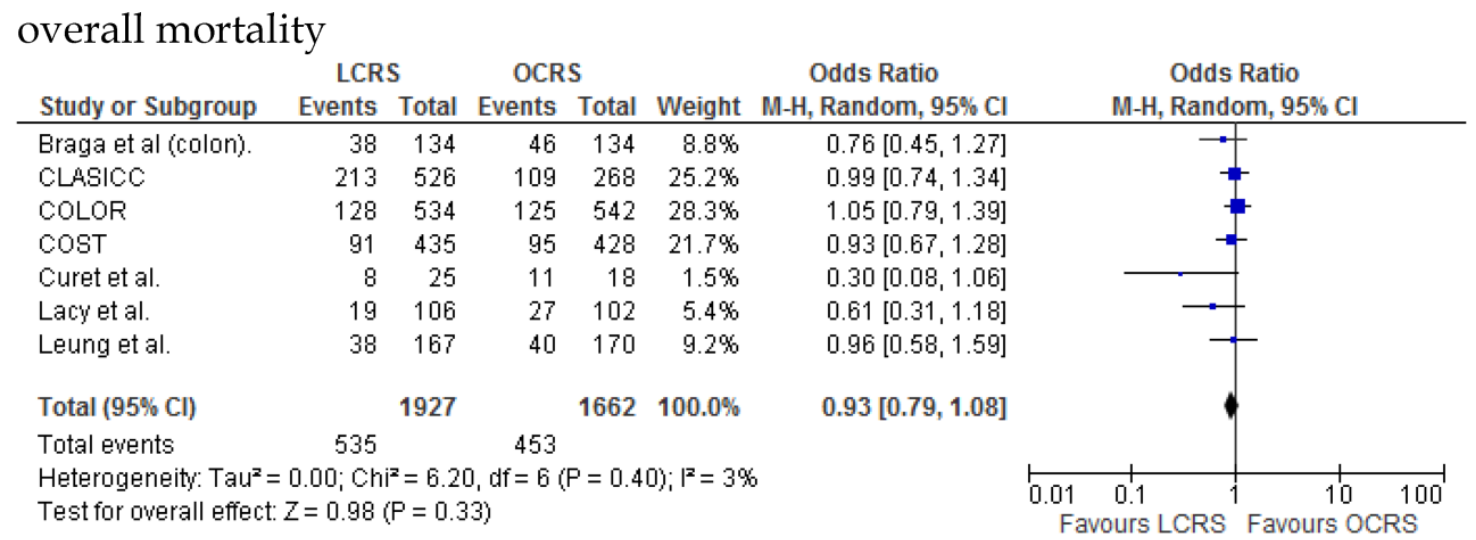

Fig. 2 Meta-analysis of the long- term period for colorectal cancer

\section{Discussion}

Previous articles showed that laparoscopic surgery for CRC is associated with low morbidity, less pain, fast recovery, and short hospital stay, compared to conventional open surgery in the short-term ${ }^{5-7}$. Recent articles reporting RCTs have shown that long-term oncological results for LCRS are comparable to those for OCRS ${ }^{33}$. There are claims that LCRS prolongs cancer-related survival ${ }^{10}$. Therefore, we examined the oncological results of LCRS and compared to those of OCRS in short- and long-term periods by a meta-analysis of 12 RCTs.

In the short-term period, this meta-analysis showed that LCRS has a significantly long operative time but significantly reduces the intraoperative blood loss compared with OCRS. These results are consistent with those of the recent RCTs ${ }^{10,22}$. Potential explanations for the abovementioned results are meticulous dissection facilitated by instruments for laparoscopic surgery and videoscopic magnification ${ }^{34}$. We also found that there was no significant difference in the number of patients requiring blood transfusions between the LCRS and OCRS groups. The lack of difference in the number of harvested lymph nodes between the 2 groups may suggest that the quality of the operative techniques is the same. Patients who underwent LCRS resumed oral intake significantly earlier and had significantly shorter hospital stays than did patients who underwent OCRS; this finding suggests that LCRS leads to faster recovery. The safety and feasibility of LCRS is similar to that of OCRS as shown by insignificant differences in the overall perioperative complications, anastomotic leakage, and perioperative mortality between the surgery groups.

In the long-term period, this study showed that there is no significant difference in the overall recur- rence, local recurrence, or distant recurrence of metastases between the LCRS and OCRS groups. The rate of wound-site recurrence for the LCRS group was significantly higher than that for the OCRS group. In 7 of the 9 studies that reported data on wound-site recurrence, the rates of wound-site recurrence for LCRS were similar to the rates for OCRS. In the CLASICC trial, the number of extraction-site recurrences was higher than that of trocar-site recurrences in the LCRS group. Therefore, the authors emphasize the need for adequate wound protection during specimen extraction $^{13}$. In the COLOR trial, the number of trocar-site recurrences was higher than that of extraction-site recurrences in the LCRS group. In this meta-analysis the differences of wound-site recurrence between the groups was insignificant, restricting wound-recurrence to isolated abdominal-wall recurrences, in the absence of recurrent disease elsewhere. Lim et al. reported that port-site metastasis may be a part of the systemic disease rather than an unfortunate sequelae of the learning curve for laparoscopic surgery ${ }^{35}$.

We found no significant difference between the LCRS and OCRS groups for overall mortality, 3- and 5-year disease-free survival rate and cancer-related mortality. These results suggest that the long-term oncological results of LCRS are similar to those of OCRS. Lacy et al. reported that LCRS significantly prolongs cancer-related survival in treatment of colon cancer ${ }^{10}$, but our meta-analysis of the pooled data did not show this difference.

Quality of life (QOL) after laparoscopic surgery is improved in the early postoperative period compared with QOL after open surgery. In the long-term period, however, QOL after LCRS is similar to QOL after OCRS9, 36. From the cosmetic viewpoint, LCRS is superior to OCRS because the length of operation 
wound was significantly shorter in LCRS than in OCRS.

Significant heterogeneity was observed between the 12 RCTs for intraoperative blood loss, duration of hospital stay, length of operation wound, overall complications in the short-term period, and overall mortality in the long-term period. This heterogeneity may be attributable to variation in the skills of the surgeons and the condition of the tumor.

In conclusion, this meta-analysis showed that LCRS has the benefits of reducing intraoperative blood loss, earlier resumption of oral intake, and shorter duration of hospital stay in short-term and seems to be similar in the long-term oncological outcomes, comparing to OCRS. Therefore LCRS may be an acceptable treatment as OCRS for CRC.

\section{Conflict of Interest}

The authors have declared that no conflict of interest exists.

\section{References}

1. [Internet] WHO. http://www.who.int/mediacentre/ factsheets/fs297/en/

2. [Internet] National Cancer Institute. http://www.cancer.gov/ cancertopics/types/commoncancers

3. [Internet] Cancer Research UK. http://info.cancerresearchuk. org/cancerstats/types/bowel/incidence/

4. Jacobs M, Verdeja JC, Goldstein HS. Minimally invasive colon resection (laparoscopic colectomy). Surg Laparosc Endosc. 1991; 1: 144-150.

5. Schwenk W, Böhm B, Müller JM. Postoperative pain and fatigue after laparoscopic or conventional colorectal resections. A prospective randomized trial. Surg Endosc. 1998; 12: 1131-1136.

6. Abraham NS, Young JM, Solomon MJ. Meta-analysis of short-term outcomes after laparoscopic resection for colorectal cancer. Br J Surg. 2004; 91: 1111-1124.

7. Gao F, Cao YF, Chen LS. Meta-analysis of short-term outcomes after laparoscopic resection for rectal cancer. Int J Colorectal Dis. 2006; 21: 652-656.

8. Jayne DG, Thorpe HC, Copeland J, et al. Five-year follow-up of the Medical Research Council CLASICC trial of laparoscopically assisted versus open surgery for colorectal cancer. Br J Surg. 2010; 97: 1638-1645.

9. Jayne DG, Guillou PJ, Thorpe H, et al. Randomized trial of laparoscopic-assisted resection of colorectal carcinoma: 3-year results of the UK MRC CLASICC Trial Group. J Clin Oncol. 2007; 25: 3061-3068.

10. Lacy AM, García-Valdecasas JC, Delgado S, et al. Laparoscopy-assisted colectomy versus open colectomy for treatment of non-metastatic colon cancer: a randomised trial. Lancet. 2002; 359: 2224-2229.

11. Araujo SE, da Silva eSousa AHJr, de Campos FG, et al. Conventional approach $\mathrm{x}$ laparoscopic abdominoperineal resection for rectal cancer treatment after neoadjuvant chemoradiation: results of a prospective randomized trial. Rev Hosp Clin Fac Med Sao Paulo. 2003; 58: 133-140.

12. Braga M, Frasson M, Zuliani W, et al. Randomized clinical trial of laparoscopic versus open left colonic resection. Br J Surg. 2010; 97: 1180-1186.
13. Braga M, Frasson M, Vignali A, et al. Laparoscopic resection in rectal cancer patients: outcome and cost-benefit analysis. Dis Colon Rectum. 2007; 50: 464-471.

14. Braga M, Vignali A, Zuliani W, et al. Laparoscopic versus open colorectal surgery: cost-benefit analysis in a single-center randomized trial. Ann Surg. 2005; 242: 890-896.

15. Braga M, Frasson M, Vignali A, et al. Laparoscopic vs. open colectomy in cancer patients: long-term complications, quality of life, and survival. Dis Colon Rectum. 2005; 48: 2217-2223.

16. Guillou PJ, Quirke P, Thorpe H, et al. Short-term endpoints of conventional versus laparoscopic-assisted surgery in patients with colorectal cancer (MRC CLASICC trial): multicentre, randomised controlled trial. Lancet. 2005; 365: 1718-1726.

17. Buunen M, Veldkamp R, Hop WC, et al. Survival after laparoscopic surgery versus open surgery for colon cancer: long-term outcome of a randomised clinical trial. Lancet Oncol. 2009; 10: 44-52.

18. Veldkamp R, Kuhry E, Hop WC, et al. Colon cancer Laparoscopic or Open Resection Study Group (COLOR). Laparoscopic surgery versus open surgery for colon cancer: short-term outcomes of a randomised trial. Lancet Oncol. 2005; 6: 477-484.

19. Clinical Outcomes of Surgical Therapy Study Group. A comparison of laparoscopically assisted and open colectomy for colon cancer. N Engl J Med. 2004; 350: 2050-2059.

20. Curet MJ, Putrakul K, Pitcher DE, et al. Laparoscopically assisted colon resection for colon carcinoma: perioperative results and long-term outcome. Surg Endosc. 2000; 14: 1062-1066.

21. Leung KL, Kwok SP, Lam SC, et al. Laparoscopic resection of rectosigmoid carcinoma: prospective randomised trial. Lancet. 2004; 363: 1187-1192.

22. Liang JT, Huang KC, Lai HS, et al. Oncologic results of laparoscopic versus conventional open surgery for stage II or III left-sided colon cancers: a randomized controlled trial. Ann Surg Oncol. 2007; 14: 109-117

23. Mirza MS, Longman RJ, Farrokhyar F, et al. Long-term outcomes for laparoscopic versus open resection of nonmetastatic colorectal cancer. J Laparoendosc Adv Surg Tech A. 2008; 18: 679-685.

24. Park IJ, Choi GS, Lim KH, et al. Laparoscopic resection of extraperitoneal rectal cancer: a comparative analysis with open resection. Surg Endosc. 2009; 23: 1818-1824.

25. Kaiser AM, Kang JC, Chan LS, et al. Laparoscopic-assisted vs. open colectomy for colon cancer: a prospective randomized trial. J Laparoendosc Adv Surg Tech A. 2004; 14: 329-334.

26. Stage JG, Schulze S, Møller P, et al. Prospective randomized study of laparoscopic versus open colonic resection for adenocarcinoma. Br J Surg. 1997; 84: 391-396.

27. Milsom JW, Böhm B, Hammerhofer KA, et al. A prospective, randomized trial comparing laparoscopic versus conventional techniques in colorectal cancer surgery: a preliminary report. J Am Coll Surg. 1998; 187: 46-55.

28. Zhou ZG, Hu M, Li Y, et al. Laparoscopic versus open total mesorectal excision with anal sphincter preservation for low rectal cancer. Surg Endosc. 2004; 18: 1211-1215.

29. Winslow ER, Fleshman JW, Birnbaum EH, et al. Wound complications of laparoscopic vs open colectomy. Surg Endosc. 2002; 16: 1420-1425.

30. Braga M, Frasson M, Vignali A, et al. Laparoscopic vs. open colectomy in cancer patients: long-term complications, quality of life, and survival. Dis Colon Rectum. 2005; 48: 2217-2223.

31. Kuhry E, Schwenk WF, Gaupset R, et al. Long-term results of laparoscopic colorectal cancer resection. Cochrane Database Syst Rev. 2008;:CD003432.

32. DerSimonian R, Laird N. Meta-analysis in clinical trials. Control Clin Trials. 1986; 7: 177-188.

33. Ohtani H, Tamamori $Y$, Azuma T, et al. A Meta-analysis of the Short- and Long-Term Results of Randomized Controlled Trials 
That Compared Laparoscopy-Assisted and Conventional Open Surgery for Rectal Cancer. J Gastrointest Surg. 2011; [Epub ahead of print]

34. Bonjer HJ, Hop WC, Nelson H, et al. Transatlantic Laparoscopically Assisted vs Open Colectomy Trials Study Group. Laparoscopically assisted vs open colectomy for colon cancer: a meta-analysis. Arch Surg. 2007;142: 298-303.

35. Lim SW, Cho SH, Oh BR, et al. Port-site recurrence after laparoscopy-assisted low anterior resection: the sign of peritoneal dissemination. Int J Colorectal Dis. 2010 [Epub ahead of print].

36. Dowson H, Cowie A, Ballard K, et al. Systematic Review of Quality of Life following Laparoscopic and open colorectal surgery. Colorectal Dis. 2008; [Epub ahead of print]. 